\title{
¿Ciudadanía generizada? AlCANCES y limitaciones DE LAS POLÍTICAS DE GÉNERO EN SUDÁFRICA POST-APARTHEID
}

\author{
Mónica Inés Cejas
}

Resumen: En este trabajo se analiza la construcción tensa de un nuevo concepto de ciudadanía -inclusiva, no racista y no sexista-en Sudáfrica a partir del proceso de transición democrática que se inicia con el fin del apartheid en la década de 1990, focalizando en la ciudadanía de las mujeres.

Palabras clave: Sudáfrica, género, ciudadanía, apartheid, post apartheid.

Enviado a dictamen: 03 de septiembre de 2008. Aprobación: 18 de noviembre de 2008.

Dra. Mónica Inés Cejas, Doctora en Estudios Internacionales y Culturales por la Universidad Tsuda de Tokio (Japón), Maestría en Estudios de Asia y África por El Colegio de México y en Estudios Internacionales y Culturales por la Universidad Tsuda, Tokio, Japón, actualmente profesora investigadora en el Departamento de Política y Cultura de la Universidad Autónoma Metropolitana-Xochimilco, Programa de Especialización y Maestría en Estudios de la Mujer. Directora de la revista Política y Cultura del mismo departamento que es parte del padrón de excelencia de CONACYT, líneas de investigación: género, movimientos de mujeres y feminismos en África; nación y ciudadanía, racismo y otras formas de otredad. Políticas de la memoria en Sudáfrica y Rwanda, correo electrónico: monicacejas@gmail.com.
Abstract: In this paper I will analyze the fraught construction of new citizenship concept-inclusive, non-racial, non-sexist-in South Africa after apartheid-the so called democratictransition-focusing on women's citizenship.

Key words: South Africa, gender, citizenship, apartheid, post apartheid.

$\mathrm{E}$ 14 de febrero de 2007, la ONG Género y Comercio en África (GENTA, por sus siglas en inglés) ${ }^{1}$ publicó una carta abierta al entonces presidente, Thabo Mbeki, como respuesta a su informe presidencial del 9 de febrero. ${ }^{2}$ GENTA critica, en este documento, la falta de políticas concretas para mejorar las condiciones de vida de la mayoría de las africanas en Sudáfrica después de más de 10 años de "transición democrática". Con una postura discursiva que recurre a la ciudadanía como figura identitaria para legitimar los reclamos que se despliegan a lo largo del documento, esta carta es una muestra clara de la construcción tensa de un nuevo concepto de ciudadanía post apartheid que debe generizarse (es decir que debe incluir en su formulación al discurso de género) para ser "políticamente correcto" $y$, a la vez, ser un nuevo referente para la acción política de los grupos excluidos durante el régimen de apartheid. 
En efecto, de acuerdo con el discurso de GENTA, la "nueva" noción de ciudadanía, definida claramente en la Constitución sudafricana de 1996, sigue instalada en el plano propositivo, de allí que se aluda a las expectativas que generaba el mensaje presidencial al que responden -que marca la mitad exacta del mandato de Mbeki 2004-2009-3 respecto a la posibilidad de "avanzar en nuestra ciudadanía como mujeres sudafricanas". ${ }^{4} \mathrm{Y}$ es a partir de este posicionamiento en una ciudadanía inclusiva, no racista y no sexista que el documento de GENTA muestra las fisuras del discurso presidencial. Con esto también se cuestionan los resultados reales de las políticas de género más impresionantes del continente a nivel formal.

En este trabajo propongo explorar desde una perspectiva de género el proceso de reconfiguración del concepto de ciudadanía post apartheid partiendo de las características del mismo durante el régimen para volver luego a los argumentos de la carta abierta de GENTA.

\section{Ciudadanía durante y después del apartheid. Algunas consideraciones sobre ciudadanía y género}

Cuenta tenida del carácter fundacional de las epistemologías clásicas del liberalismo, que asumen a la ciudadanía como estatus, y el republicanismo que la ve más bien como práctica, la crítica feminista (sobre todo en Carole Pateman ${ }^{5}$ ) ha partido de su deconstrucción para develar la metanarrativa que descentra a las mujeres de los espacios de poder. Demuestra que la racionalidad de los autores del derecho ha sido también una construcción social, y con esto la falsedad de la supuesta universalidad de la ciudadanía (lo universal es generado desde espacios hegemónicos y en ventaja que imponen lo que en realidad es una visión parcial a los otros). La crítica feminista en teoría política ha afectado el análisis tradicional de la ciudadanía proponiendo una lectura crítica del pensamiento clásico, al discutir la dicotomía público-privado desde una perspectiva de género y redefinir las ideas de igualdad y diferencia. De acuerdo a Pateman, el contrato social es ante todo un pacto social-sexual patriarcal (o fraternal en el caso del estado liberal), ciego a medias hacia aquellos sectores de la población que no pueden acceder al espacio público para demostrar su participación en los asuntos del estado mediante el ejercicio de sus derechos. En este ejercicio Pateman rebela además la aporía de la nación: el esencialismo universalista homogeneizante basado en una producción y reproducción de la diferencia (producida por la negación de la misma en la formulación de modelos ideales). En efecto, el liberalismo considera al "individuo" como unidad básica de la vida democrática argumentando por un igualitarismo en derechos con independencia de la riqueza, el estatus, la raza, el sexo, etcétera, y en el que, por tanto, no cuentan las diferencias de ninguna clase; todos somos "ciudadanos iguales" aunque los patrones bajo los cuales se definió a ese individuo correspondan a modelos hegemónicos que niegan la diferencia (excluyendo histórica y políticamente a las mujeres o a determinados grupos étnicos, por ejemplo) y al hacerlo la instauran mediante prácticas políticas que se basan precisamente en una diferenciación clara entre los miembros de la sociedad (por ejemplo en su desigual ejercicio legítimo de los derechos por género, grupo étnico, nivel de escolaridad, etc.). ${ }^{7}$ El universalismo también niega que las maneras en que se experimenta y percibe esa ciudadanía también sean plurales. ${ }^{8}$ Pero además, el liberalismo define la esfera pública haciendo abstracción de la privada conceptualizada como locus natural de las mujeres cuya "intimidad" debe ser protegida del control tiránico del estado. ${ }^{9}$ El republicanismo también apela a las nociones de universalismo e imparcialidad por "el bien común"10 pero insistiendo más bien en la práctica ciudadana. En ambos se asume una determinada división sexual del trabajo y por lo tanto la (im) posibilidad de acceso a los recursos y a los espacios de poder. Si los derechos de ciudadanía fueron definidos centralmente a partir de un sujeto con características determinadas, no sólo se excluyó a quienes no poseían las mismas, sino que esos derechos no corresponden a las 
diferencias ni satisfacen las necesidades de otros grupos. Según Pateman ${ }^{11}$ la exclusión de las mujeres era parte del "acuerdo" del proyecto liberal después de la Revolución Francesa: una fraternidad en la que los hombres hacían un contrato de igualdad entre ellos mismos y como parte de este acuerdo obtenían el derecho de gobernar a las mujeres en la esfera privada; los hombres se volvían "representantes de la familia". Por lo tanto, la ciudadanía se convirtió en un modelo masculino en el que los "atributos femeninos" son la excusa para la exclusión de las mujeres. Para Pateman se requeriría de un "concepto sexualmente diferenciado de ciudadanía" que le otorgara un significado político a las capacidades de las mujeres, "incluyendo a las mujeres como mujeres" en un contexto de igualdad civil y ciudadanía activa. La salida para algunas fue re-imaginar la ciudadanía y la esfera pública para que diera cabida a los "valores femeninos" al resaltar las cualidades superiores maternales de cuidado, responsabilidad y compasión como constitutivos claves de la ciudadanía. Según Dietz, "las feministas maternalistas" se basan en la idea de una conciencia política femenina fundada en las virtudes de la esfera privada de las mujeres, principalmente en la maternidad. Las "maternalistas" desean establecer la primacía moral de la familia y al reconsiderar la distinción liberal entre privado y público, consideran lo privado como un "lugar para una posible moral pública y como un modelo para la actividad de la ciudadanía misma" ${ }^{\text {"2 }}$. El riesgo está en que al hacerlo se instaura otro tipo de esencialismo que convierte a "la mujer" en entidad universal y ahistórica. Elénfasis en la ciudadanía diferenciada ha sido acogido por multiculturalistas ${ }^{13}$ y otras feministas (las ya mencionadas Young y Lister, por ejemplo) quienes plantean un universalismo diferenciado que, sin abandonar el ideal universal implícito en la ciudadanía, busca tematizar e incluir las diferencias, o bien un diálogo transversa ${ }^{14}$ que parta de la multiplicidad que implica la diferencia.

¿Pero qué ocurre si trasladamos el concepto y las nociones de ciudadanía a otro contexto diferente al de occidente? En el caso de las sociedades africanas, corresponden en origen a una experiencia histórica ajena, la europea, en sus diversas expresiones y con diferentes legados organizativos a nivel socioeconómico, político-jurídico, territorial, etcétera. ${ }^{15}$ Durante el período colonial, el espacio social dentro del cual una sociedad podría volverse "civil" en África fue bloqueado. Mediante artilugios legales, el colonizado fue un "sujeto" (masculino) con derechos políticos y sociales altamente circunscriptos. Económicamente, el sujeto colonial masculino era una unidad de trabajo, un "homme adulte valide" tal como aparecía categorizado en el censo colonial belga. Culturalmente, el dominio de los africanos requería un sistema clasificatorio establecido desde fuera. Se trató de un África "inventada", habitada por el "otro subordinado" en un proceso de construcción social de identidad marcado por políticas y prácticas coloniales. Como señala Mudimbe, mediante su dominio del espacio físico y del discurso, el estado colonial "transformó áreas no-europeas en fundamentalmente constructos europeos". ${ }^{16} \mathrm{Y}$ es por eso que la noción de ciudadanía surgida de esta práctica colonial, como afirma Patricia Mcfadden, ${ }^{17}$ identificó al estatus de ciudadano con el hombre blanco propietario (expresado por mucho tiempo en el sufragio calificado). Las mujeres de color totalmente invisibilizadas en este discurso serán relegadas al espacio rural colonial (considerado "auténticamente africano" y bajo el gobierno de los jefes y ancianos) o admitidas en el urbano sólo en calidad de esposas.

En los años finales del estado colonial, una intensa voz de protesta comenzó a emerger con planteamientos nacionalistas. Se cristalizaron formas más activas de acción cívica —-movimientos nacionalistas, actividades de asociación- acompañadas por otras formas de conciencia colectiva, que reflejaban las alteraciones en las estructuras social y comunal producidas por la experiencia colonial. El Estado poscolonial debía borrar "lo tradicional" y servir como "pedagogo de la modernidad". Las mujeres participaron en estas luchas y con ello comenzaron a producir otra noción de ciudadanía: 
como sujetos autónomos, conscientes de sus derechos. La paulatina participación de las mujeres en el espacio público se fue produciendo no sin tensiones ya que el estado moderno intentaba reproducir el modelo occidental de separación público-privado, por lo que la lucha por la definición de un tipo de ciudadanía inclusiva, no sexista y no racial después de las independencias, se trasladó al plano constitucional y jurídico. De allí que las luchas se concentrasen en la derogación de aquellas leyes heredadas de la colonia que restringían la movilidad de las mujeres en el espacio urbano. Con esto se ponía en discusión qué es lo público y qué lo político; se redefinía la relación con el estado al exigir el reconocimiento de los derechos que liberaban a las mujeres de su condición de eterna menor: acceso a la propiedad, a la patria potestad sobre sus hijos, derechos sexuales reproductivos y los que la agenda a nivel internacional (sobre todo a partir de la Conferencia de Beijing en 1995) fue generando, en una dinámica local-global de los movimientos de mujeres. ${ }^{18}$

\section{Mujeres sin derechos ciudadanos durante el apartheid ${ }^{19}$}

La posición de las mujeres durante el régimen de apartheid era producto de relaciones de clase, racismo y subordinación de género. Factores que establecieron privilegios y subordinaciones no sólo entre géneros sino entre las mismas mujeres. De allí que las mujeres hayan experimentado su posición estructural de manera diferente a los hombres. De todas ellas, las africanas fueron las más excluidas al negárseles cualquier derecho que podamos identificar con ciudadanía. ${ }^{20}$ Aún antes de la institucionalización del apartheid y desde el punto de vista de los administradores blancos, las peticiones y protestas sólo podían ser aceptadas cuando estaban lideradas por un hombre. En efecto, cuando las mujeres organizaban protestas de gran magnitud, la política típica de los administradores era reunir a los hombres y reclamarles "el control de sus mujeres". ${ }^{21} \mathrm{El}$ sistema legal instituido con el colonialismo y la transición al capitalismo profundizaron el régimen patriarcal ya existente en las sociedades tradicionales. Así, la propiedad privada fue definida de manera que las mujeres fueron totalmente excluidas del acceso a la misma. Bradford señala por ejemplo, que el "Natal Code" de 1891 (la fijación en letra escrita de las leyes de costumbre como política de la administración colonial británica) instituía que los ingresos de las mujeres pertenecían a los hombres bajo cuya tutela permanecían de por vida: padre, hermano, esposo o hijo. ${ }^{22}$ Las mujeres eran consideradas perpetuas menores que debían estar sujetas a la autoridad masculina toda la vida. La sanción de la Native Administration Act en 1927 que oficializó la aplicación de la ley de costumbre en los casos civiles entre africanos y a la vez aumentó el poder de los jefes tradicionales (quienes pasaron a mediar entre la administración general y las comunidades como una elemento más del aparato burocrático estatal) afianzó el afincamiento de las africanas como menores.

Respecto a las regulaciones del movimiento de población bajo criterios raciales, pilar del régimen de apartheid, puede afirmarse que hasta la década de 1950 las mujeres africanas no fueron directamente afectadas por las leyes de pases que desde 1923 se aplicaban a los hombres. Y aunque los intentos por controlar el movimiento de las mujeres comenzaron tempranamente en Orange Free State a principios del siglo XX, las protestas de las mujeres consiguieron disminuir sus efectos y su extensión a otras partes del país. Sin embargo, y como señala Joanne Yawitch, ${ }^{23}$ ciertas regulaciones establecidas en la década de 1930 crearon un campo propicio para el control de la movilidad de las mujeres. Por ejemplo, según el "amendment to the Urban Areas Act" el estatus urbano de las mujeres pasó a depender de la posibilidad de probar que un pariente masculino contase con residencia urbana establecida. En 1937 se agregó otro requerimiento, el de tener un lugar de residencia "adecuado". Pero las implementaciones no fueron estrictas, lo que explica el crecimiento sostenido de la cantidad de mujeres viviendo en el medio urbano desde esa época. 
En 1948 con el establecimiento de la administración del Partido Nacional comenzó a presionarse para extender el control sobre las mujeres. Los nacionalistas consideraban que no era conveniente crear un proletariado urbano estable. A cambio proponían un sistema de migraciones más sofisticado, es decir, un sistema que evitase el establecimiento urbano permanente de la mano de obra sobrante, sobre todo femenina. El objetivo era que volviesen a las reservas y sostuviesen la economía de las mismas, a la vez que aseguraban la reproducción de la mano de obra. En 1952 con la llamada Abolition of Passes and Coordination of Documents, el sistema de control de identidad de la población masculina africana fue unificado en un solo documento: "the reference book". Elmismo contenía información detallada sobre el lugar de residencia legal, trabajo, pago de tasas y, de ser necesario, el permiso para permanecer en zonas urbanas. ${ }^{24}$ Desde 1958 y no sin resistencias, comenzó a requerirse la posesión de "reference books" ("dompas") como condición de empleo para las mujeres africanas. Desde la década de 1960 si perdían su empleo o enviudaban debía salir de la zona urbana y regresar a las reservas a menos que pudiesen probar residencia legal por más de 15 años. Para obtener un "reference book" era necesario probar que se había nacido en el lugar o que se vivía allí desde que el sistema de permisos fue establecido. En el caso de una mujer soltera era necesario probar que alguno de sus padres contaba con permiso de residencia o tener un contrato de trabajo con algún empleador. Si sus padres morían o terminaba su contrato, cesaba el permiso para permanecer en el área. Sólo podría permanecer si se casaba con un hombre con permiso de residencia. Las mujeres sin "reference books" no podían rentar casa en las áreas urbanas, o perdían las que tenían si no se registraban. No podían registrar el nacimiento de sus hijos o casarse de acuerdo a la "common law". Tampoco podrían recibir jubilaciones o pensiones en su vejez. Las docentes y enfermeras serían despedidas de sus trabajos. También sería necesario presentar un "reference book" para obtener el permiso para ejercer el comercio informal y pagar rentas.
La ley de pases se abolió en 1986 así como la que prohibía los matrimonios mixtos (Mixed Marriages Act de 1949), sin embargo fue recién en 1991 cuando se abolieron las leyes que instituían el registro obligatorio de población con base en criterios raciales (Group Areas and Population Registration Acts).

\section{Ciudadanía post apartheid}

Aunque las fechas emblemáticas y "oficiales" que marcan el "fin" del apartheid son el 2 de febrero de 1990 (discurso del entonces presidente F.W. de Klerk al declarar el fin del sistema) y el 27 de abril de 1994 (primeras elecciones universales $)^{25} \mathrm{el}$ proceso de cambio institucional-legal comenzó en diciembre de 1993, después de dos años de intensas negociaciones cuando el Parlamento sudafricano dio fin a la dominación política de la minoría blanca sancionando una Constitución interina. ${ }^{26}$ Se abrieron así las puertas al primer gobierno no-racial en Sudáfrica. La Constitución interina, si bien resultó de negociaciones, puede considerarse el producto de los principios y aspiraciones de la lucha de liberación expresada en manifiestos como la Cartade las Mujeres (Women's Charter) de 1954, ${ }^{27}$ la Carta de la Libertad (Freedom Charter) de 1955, los principios constitucionales del ANC en 1988 y el borrador de la declaración de derechos del ANC en 1990. Especialmente la Carta de la Libertad ${ }^{28}$ fue una respuesta de los sectores oprimidos por el apartheid al presentar un modelo de sociedad futura unida y democrática. El concepto de igualdad expresado en estos documentos combina la igualdad económica y la política priorizando a la primera. Se insiste en que para lograr una verdadera liberación no basta con la igualdad en derechos y oportunidades (derechos políticos y civiles) sino que es indispensable la transformación de las condiciones económicas (mediante la justicia social y la redistribución de los recursos) que sustentan las desigualdades política, social y económica del apartheid.

La Constitución interina y la sancionada en $1996^{29}$ se basan en los principios de la democracia liberal y el 
constitucionalismo al establecer el sufragio universal, un Parlamento conformado por una Asamblea Nacional y un Consejo de Provincias, un gobierno central fuerte con nueve gobiernos regionales, un sistema judicial independiente y una declaración de derechos. En sus textos se garantiza la igualdad de género y se crea un conjunto de instituciones que aseguren que la inclusión de una perspectiva género, como más que agregado, sea efectiva en el gobierno y el sector privado. Al ser el derecho de igualdad piedra angular de la Constitución, todos los derechos deben ser interpretados con miras a hacer efectiva la igualdad-el derecho a la igualdad debe ser sustantivamente interpretado- Todo esto en un discurso donde la igualdad de género y los derechos sociales aparecen como constitutivos esenciales del nuevo concepto de ciudadanía sudafricana. ${ }^{30}$ Desde la sanción de la Constitución interina se produjo entonces una ruptura con el pasado de apartheid porque se estableció una estructura legal para un estado democrático comprometido con los derechos humanos. Para operacionalizar este cometido, la Constitución fijaba determinadas responsabilidades en el gobierno para atender las desigualdades socioeconómicas como política esencial para combatir las desigualdades de género y las basadas en criterios racistas. ${ }^{31}$

La nueva Constitución aprobada en 1996 (Act 108) 32 dejó establecidos los principios primordiales del funcionamiento social:

- Igualdad, dignidad humana y libertad

- Aparato legal no sexista y no racista

- Respeto a los derechos humanos

- Consideración del derecho internacional (debe ser tenido en cuenta en los tribunales)

- Sufragio adulto universal, sistema multipartidista

Existe desde entonces una ciudadanía inclusiva común sudafricana por la que todos los ciudadanos tienen igual acceso a los derechos, privilegios y beneficios, y son igualmente sujetos a los deberes y responsabilidades de la ciudadanía. Los siguientes artículos apuntan específicamente, a una ciudadanía inclusiva de género en la medida en que abarcan todos aquellos derechos y garantías que asegurarían la equidad en una sociedad post apartheid.

Respecto a la relación entre igualdad y discriminación el artículo 9, capítulo 2, inciso 3, declara:

El estado no puede discriminar directa o indirectamente contra una persona en base a una o más de las siguientes premisas: raza, género, sexo, embarazo, estado civil, origen étnico o social, color, orientación sexual, edad, discapacidad, religión, conciencia, creencia, cultura, lenguaje y nacimiento.

\section{Libertad y seguridad de la persona}

Capítulo 2, inciso 12 (2). Todos tienen derecho a la integridad física y psicológica, que incluye los derechos a:

- Tomar decisiones concernientes a la reproducción

- Tener seguridad sobre y controlar el propio cuerpo.

- No ser sujeto a experimentos científicos sin previo consentimiento.

\section{Libertad de religión, creencia y opinión:}

Capitulo 2, inciso 15 (3) por el que se reconoce que ciertas prácticas religiosas o consuetudinarias pueden ser reconocidas, pero siempre y cuando sean consistentes con la Constitución.

\section{Libertad de expresión:}

Capítulo 2, inciso 16 (2) (c) el derecho de libertad de expresión no incluye las acciones que promueven el odio en base a criterios de raza, etnicidad, género o religión. 
Y además los derechos de propiedad (Cap. 2, inciso 25), a una vivienda adecuada (Cap. 2, inciso 26); acceso a servicios de salud incluyendo los de salud reproductiva, agua y alimentación suficiente y seguridad social (Cap. 2, inciso 27); a la educación básica incluyendo la educación básica de adultos (Cap. 2, inciso 29); al lenguaje y cultura de elección siempre (inciso 30); de las comunidades culturales, religiosas y lingüísticas a disfrutar de su cultura, hacer uso de su lengua y practicar su religión y a formar, incorporarse y mantener asociaciones (inciso 31); acceso a las cortes (inciso 34).

A nivel institucional se estableció lo que se dio en llamar “A Nacional Machinery for Women”, es decir, estructuras coordinadas dentro y fuera del gobierno con el objetivo de lograr la equidad de género que se implementaron desde 1996-97 basadas en el principio de transversalidad (gender mainstreaming). ${ }^{33} \mathrm{Y}$ fue entonces cuando en 1996 se insalaron dentro de la legislatura un grupo multipartidario de mujeres parlamentarias y un comité para el mejoramiento de la calidad de vida y estatus de las mujeres. Este último quedaría a cargo del monitoreo del cumplimiento de las disposiciones de CEDAW (Convención sobre la eliminación de todas las formas de Discriminación contra la mujer) así como del análisis del presupuesto nacional con una perspectiva de género. En el seno del poder ejecutivo (dependiente de la oficina del vicepresidente con contrapartes a nivel provincial) se creó en 1997 una Oficina por el Estatus de las Mujeres (Office for the Status of Women, OSW) cuya misión es coordinar y facilitar la implementación de las políticas y programas de género del gobierno. ${ }^{34}$ El gabinete presidencial aprobó en 1996 el establecimiento de "focos de género" en todos los departamentos del gobierno. Finalmente se estableció también un cuerpo independiente: la Comisión para la Igualdad de Género (Comisión on Gender Equality, CGE) en 1997, la que formada por 11 comisionados:

1. Debe promover el respeto por la igualdad de género y la protección, desarrollo y logro de la igualdad de género.
2. Tieneel poder necesario, regulado por la legislación nacional, parallevar a cabo sus funciones, incluyendo monitoreo, investigación y educación. También funge como aparato consultivo de las políticas de gobierno y puede presentar reportes en cuestiones concernientes a la igualdad de género.

\section{Fisuras y tensiones}

Este panorama, aparentemente brillante, queda ensombrecido si se tiene en cuenta que no logra borrar las desigualdades aún pendientes:

- La persistencia de niveles inferiores en los salarios de las mujeres en comparación con los de los hombres en un contexto (datos de 2003, Labour Force Survey, Statistics South Africa, Pretoria) donde las mujeres sudafricanas constituyen $42 \%$ de la fuerza de trabajo pero sólo 30\% de los empleados formales y $51 \%$ de los desempleados (situación mucho más grave en las mujeres menores de 30 que constituyen $75 \%$ de los desempleados). ${ }^{35}$

- La situación de las mujeres de las zonas rurales donde constituyen la categoría de los ciudadanos más pobres del país. Sobre todo en los ex bantustanes o homelands donde las mujeres son $56 \%$ de la población (frente a 52\% para el resto del país) y donde $81 \%$ percibe ingresos mensuales inferiores a los 1,000 rands. ${ }^{36}$ Con un programa de reforma agraria formulado de manera ambiciosa pero que demora en implementarse de manera dinámica debido a "la falta de capacidad institucional" (justificación característica durante la década de 1990 según Walker $^{37}$ ) para procesar los más de 23000 juicios de reclamo y sin lineamientos claros para asegurar la inclusión de las mujeres en los procesos de redistribución y restitución de tierras y reforma de la tenencia. A esto se 
suma la falta de acción del gobierno frente al poder que continúan teniendo las autoridades tradicionales respecto a la asignación de recursos en las comunidades rurales. Según la misma Walker: "es improbable que las mujeres pobres se beneficien de la nueva dirección que ha tomado la política de reforma agraria desde 1999, que concede prioridad a la promoción de una clase agrícola comercial negra por encima de otros compromisos" 38 en el marco de una política macroeconómica orientada hacia el inversor que limita ciertamente compromiso del gobierno de redistribución de recursos, y todo en un contexto económico social marcado por las consecuencias de políticas de discriminación en el acceso a los recursos en base a criterios racistas.

- La aplicación de la ley consuetudinaria en algunas zonas del país, que mantiene a las africanas como menores.

- Las africanas continúan siendo la principal fuerza en el sector informal de la economía.

- La violencia doméstica todavía depende de una fuerza de policía que no está suficientemente preparada para atender este tipo de casos y no cuenta con recursos suficientes.

Todo esto en un contexto exacerbado por la pandemia de VIH/SIDA con la población femenina más vulnerable a la infección ( $58 \%$ de los VIH positivo son mujeres, es decir 3.1 millones de los 5.5 millones infectados entre adultos mayores de 15 años, UNAIDS, 2006). Carentes, en muchos casos, del acceso a servicios sociales básicos (electricidad, agua potable, hospitales, etcétera) son ellas quienes tienen que hacerse cargo además, del cuidado de los enfermos de la familia que resultan VIH positivos.

¿Cómo es esto a pesar de la excelente legislación que fue descrita y un aparato institucional que aparentemente garantizaría la efectiva implementación de políticas de género? Más que una falta de recursos y capacidades en estas instituciones, para Hassim ${ }^{39}$ la clave está en combinar todo este aparato con políticas de seguridad social que, al reducir la tasa de pobreza, mejoren la autonomía personal de las mujeres y hagan efectivo su acceso a los derechos que la ciudadanía post apartheid le reconocen. Pero veamos la lectura que hace GENTA del discurso presidencial identificando las fallas del sistema.

\section{Una lectura feminista a las políticas del actual gobierno}

En su discurso presidencial ${ }^{40}$ del presente año Mbeki recurre seis veces, en su retórica, a la expresión "lo que es desagradable y repulsivo en la sociedad humana". ${ }^{41}$ Esta recurrencia sirve para marcar espacios de enunciación que articulan pasado y presente en una duración que algunas veces le permite desplazarse en el tiempo corto de su mandato (sobre todo vinculando al presente discurso con el de apertura de su segundo mandato en 2004 que coincidió con la celebración del décimo aniversario "de la liberación"); y otras veces, para incluir un tiempo de mayor duración en una perspectiva lineal y progresista de la historia. También, y al enlazar todo el discurso, la expresión permite significar y dotar de sentido ciertas subjetividades que no son definidas por su género. Esta expresión es punto de partida para señalar el apartheid como productor principal de esos contenidos cuando se aplican a la definición de lo que era Sudáfrica y también para marcar el objetivo que por encima de todos (la metanarrativa) definiría la política de su gobierno.

De acuerdo a Mbeki, durante el apartheid mientras "haber nacido negro significaba una maldición de por vida, haber nacido blanco significaba una carga de miedo permanente y furia escondida". Con esta afirmación puede conciliar a los opuestos en una retórica que llama a la inclusión bajo una misma identidad (una Sudáfrica post apartheid cuyo telos —o "sueño común" como señala Mbeki- es constituirse en "sociedad democrática, en paz, no racial y 
no sexista, próspera y comprometida con la humanidad") ya que ambos serían "victimas" aunque con un diferente significado dentro de un sistema basado en "lo desagradable y repulsivo". Y es así que son igualmente desagradables y repulsivas las condiciones de vida de los marginados por el régimen, significados en este caso por el "hedor" de la pobreza, como la falta de seguridad de los pudientes, significados en la necesidad de defensas electrificadas, altas paredes, perros gurdianes, patrullas de policía y regimientos militares para garantizar su seguridad en espacios estrictamente delimitados.

Al enlazar su presente mensaje con el discurso de 2004, Mbeki retoma lo formulado entonces como llave maestra para terminar con esta situación: la creación de fuentes de trabajo y la erradicación de la pobreza. Y esta es la antesala para afirmar la pertinencia actual de una serie de políticas de liberación de la economía (por ejemplo, la apertura a las inversiones extranjeras reduciendo sus costos de inversión). Datos cualitativos que confirmarían un progreso sostenido apoyan su discurso (tasa de crecimiento económico, número total de empleos creados, porcentaje de negros en altos puestos de gestión empresarial, etcétera). Casos pendientes como la restitución de tierras son expuestos de manera ambigua sin dejar en claro políticas que los dinamicen.

A continuación la expresión antes mencionada sirve de articulador para remarcar las direcciones de las políticas para el resto del mandato: seguridad frente al crimen; mejoramiento del sistema de políticas públicas (sobre todo a nivel de los gobiernos locales); la implementación del nuevo programa económico (AsgiSA, Accelerated and Shared Growth Initiative, Iniciativa para el cremiento compartido y acelerado) cuya máxima premisa es aumentar la inversión para mejor el nivel de competitividad de la economía; reforma del sistema de seguridad social (un impuesto para financiar los ahorros básicos para el retiro, muerte y discapacidad); políticas sociales (un subsidio al empleo para los que cuentan con salarios bajos, planes de vivienda, transporte y salud); compromisos internacionales, especialmente en el continente (papel de Sudáfrica en los procesos de pacificación en la región de los grandes lagos, Somalia, NEPAD).

La carta abierta de GENTA ${ }^{42}$ es ante todo un reclamo a la ausencia de las mujeres en la retórica de Mbeki. Y así se señala en la carta, que tal vez se refirió a ellas cuando señaló al "hedor" de la pobreza porque: "muchas de nosotras vivimos con ese hedor". Según GENTA, el presidente sólo las menciona una vez y para aludir a las "mujeres indigentes", pero se trata de mujeres sin agencia potencial porque jamás menciona "los mecanismos que les permitirían participar de manera significativa en la economía".

A sabiendas de que no se puede rebatir el discurso cuantitativo de Mbeki, GENTA reconoce el crecimiento económico de Sudáfrica y su emplazamiento como un lugar atractivo para la inversión, pero lo ataca haciendo una lectura cualitativa y de género del mismo: "este crecimiento no se traduce en el mejoramiento de las vidas de la mayoría de la gente en este país y especialmente de las mujeres quienes son la mayoría de los menos capacitados, las peor pagadas, y cuyo trabajo es el más fácil de malbaratar a los inversores extranjeros". Y es entonces que se enuncia la contradicción intrínseca entre la creación de un medio más flexible para las inversiones y la erradicación de la pobreza, si se analiza esta relación desde una perspectiva de género porque "esto significa relegar a las mujeres a los trabajos peor pagados, en condiciones frecuentemente riesgosas sin protección sindical para producir beneficios que serán repatriados al extranjero". Se trata de un proceso que termina por expulsar "lo superfluo" a su funcionamiento. Al avalar este orden, el discurso de Mbeki estaría deshumanizando a las mujeres porque objetiva a la pobreza como sitio en el que habitan.

Incluso en los casos en que en el discurso presidencial se avala por políticas de fortalecimiento de la pequeña empresa, que podrían producir un cambio efectivo en la situación de muchas mujeres, GENTA señala que si no se la acompaña con la creación de condiciones que 
habiliten el acceso de las mujeres a estas oportunidades como un entorno financiero que permita el acceso al crédito, el cuidado de los hijos, capacitación, etc., se trata de medidas que las ignoran, están vacias de sentido para este sector de la población.

En la perspectiva de GENTA el desarrollo económico necesita un enfoque de género que vaya más allá de identificar problemas sociales y económicos para "deconstruir la ideología de relaciones de género, lo que incluye la redistribución del poder". Sin un re-alineamiento radical y consistente y una redistribución de recursos que atraviese a todos los sectores junto a un cambio completo de pensamiento siempre se tratará de "aliviar" la pobreza en lugar de "erradicarla", señalan. El estado tiene que reconocer que sus políticas pueden ser negativas para los más vulnerables (y entre ellos sobre todo para las mujeres).

Otro punto importante del discurso de Mbeki fue la seguridad. GENTA llama "lapsus vergonzoso" a la omisión en el discurso del número alarmante de violaciones que hacen de Sudáfrica el país con más incidencia de violaciones en el mundo, y las otras formas de violencia de género, cuando al hablar de crimen sólo se limitó a mencionar los casos de soborno y tráfico de animales. "iEs que el soborno es de mayor prioridad que la violación, el tráfico de seres humanos y la violencia doméstica?".

Y en un ejercicio similar al de Mbeki cuando articula los tiempos de su discurso, GENTA hace retrospectiva por otros derroteros cuando trata demostrar el status quo de los programas de bienestar social, desde el GEAR (Growth, Employment and Redistribution Economy Strategy/Estrategia económica de crecimiento, empleo y redistribución) hasta el actual AsagiSA, argumentando su falta de claridad como "contrato social con la nación". Aunque el GEAR, definido por el discurso oficial como "éxitoso" con base en criterios cuantitativos - sobre todo la reducción del déficit presupuestario y de la inflación - para GENTA falló en conseguir los objetivos tal y como su nombre lo establecía. AsgiSA comparte la falta de claridad, se habla de generación de empleo, sin aclaraciones cualitativas: usted no le ha dicho a la nación que muchos de los 500,000 nuevos empleos creados son de corto tiempo o temporarios y que incluyen a personas empleadas por cuenta propia en el sector informal. Y de manera más crítica para las mujeres africanas, usted no nos ha dicho cuántos de esos empleos son para las mujeres, quienes constituyen el grupo más grande de desempleados.

\section{Reflexiones finales}

El discurso presidencial y la crítica al mismo cobran relevancia si tenemos en cuenta que el primero es parte de la narrativa oficial que produce el estado y por la que se articulan filiaciones textuales, culturales y sociales invocando "el nombre del pueblo o la nación" en un sentido incluyente para garantizar las políticas y relaciones de poder del proyecto de "nueva nación post-apartheid".

La carta de GENTA nos invita a identificar las diferencias producidas por las prácticas discursivas y los efectos discursivos de las prácticas materiales en la necesidad constante de administrar la diferencia por parte del estado. ${ }^{43} \mathrm{Y}$ es así que mediante una contranarrativa interrumpe al cuestionar la "pedagogía de la nación"44 expresada en el discurso presidencial y al hacerlo transforma los significados subjetivos y colectivos de las categorías identitarias que éste busca naturalizar.

La ciudadanía generizada, es decir transversalizada por una perspectiva de género que garantice la equidad está aún por hacerse, sobre todo si de lo que se trata es de una ciudadanía que incluya la totalidad de los derechos que aparecen en el texto constitucional, sine qua non para que la equidad no se quede a nivel propositivo, en letra muerta o reducida al ejercicio electoral. Se trata entonces de una ciudadanía que requiere agencia de parte de todos los sujetos que la animan, una participación activa en su ejercicio pleno, crítica permanente al contrato social que la sustenta y a la otra parte de ese contrato: el estado, quien a su vez debe asumir su responsabilidad en el cumplimiento del mismo. GENTA reclama entonces, 
una ciudadanía que pueda constatarse sobre todo con base en criterios cualitativos. De allí la importancia del ejercicio de identificar las trampas discursivas, tal como propone GENTA:

Es crítico, al definir una identidad nacional común, ser un conocedor de la totalidad de la nación. El carácter de la Nación-estado, Sr. Presidente, está ligado a la manera en la que el estado se relaciona con todos los que están dentro de sus fronteras. Está conectado a la nación que facilita, que proteje y que alimenta. Como ciudadanos debemos desafiar el rol del estado como protector, facilitador y defensor especialmente cuando aunque presenta todos estos roles, está vacío. Debemos, como mujeres, interrogar al discurso nacional que nos ignora o que replica lo que es reaccionario, patriarcal, ciego respecto al género, y hostil a nuestro desarrollo en el nombre del 'crecimiento' o la 'inversion' o permanece escondido bajo un escritorio de género... ${ }^{45}$ La nación en esta época requiere de un liderazgo de coraje, Sr. Presidente, que facilite la cohesión social sin amenazas a los que disienten, que nos haga sentir física, económica, social y financieramente a salvo sin tener que vender nuestros intereses al capital extranjero y que pueda vincular a las mujeres fuertes en este país más que como simples votantes el día de elecciones. ${ }^{46}$

\section{Notas}

${ }^{1}$ GENTA (Gender and Trade Network in Africa) es una red de feministas con sede en Johannesburg, Sudáfrica, que asesoran en material de comercio y género a grupos de mujeres, ONGs, movimientos sociales y oficinas gubernamentales en el continente africano. GENTA pertenece a la organización internacional IGTN (International Gender and Trade Network) que busca establecer un diálogo y estrategias de acción a nivel mundial y con una perspectiva feminista sobre comercio y globalización. En África GENTA organiza talleres sobre género, comercio, políticas de NEPAD (New Partnership for Africa's Development / Nueva Alianza para el Desarrollo de África), y WTO (World Trade Organization / Organización Mundial del Comercio) y el impacto de la globalización y la privatización en las mujeres, familias y comunidades. En GENTA participa el sector privado, los movimientos de mujeres, oficinas gubernamentales, los sindicatos, etc. Véase http://www.igtn. org/page/390

2 "State of the Nation Address of the President of South Africa, Thabo Mbeki: Joint Sitting of Parliament, February 9, 2007" en http:/www.dfa.gov.za/docs/ speeches/2007/mbek0209.htm

${ }^{3}$ El mandato de Mbeki terminó abruptamente el 20 de septiembre de 2008.

4 "A Feminist critique of president Mbeki" en Pambazuka News, 14-02-2007, en http://www.pambazuka.org/en/ category/comment/39753 (el subrayado es mío).

${ }^{5}$ Véase Pateman, Carole, The Disorder of Women, Stanford University Press, Stanford, 1989.

${ }^{6}$ Véase Young, Iris Marion, "Polity and Group Difference: A Critique of the Ideal of Universal Citizenship", en Ethics, 99, núm. 2 (enero 1989) pp. 250-274; Phillips, Anne, The Politics of Presence, Oxford, Clarendon Press, 1995 y Jelin, Elizabeth, "Igualdad y diferencia: dilemas de la ciudadanía de las mujeres en América Latina”, Ágora, No. 7, Año 3, invierno, 1997, pp. 189-213 y Las mujeres y la cultura ciudadana en América Latina, UBA-CONICET, Buenos Aires, marzo 1996, mímeo.

${ }^{7}$ Para que una persona sea elegida como autoridad requiere una mayor dedicación a la política y mayor tiempo, y con suma frecuencia son los hombres quienes pueden hacerlo o disponerlo (Phillips, 1991). Antes se requiere además una socialización determinada para la misma, algo que también tienen más los hombres; por otra parte, se necesita otra construcción cultural de la política donde se acepte de manera integral y natural la participación de la mujer del modo como se hace con la del hombre (Phillips, 1993). Véase Phillips, Anne, Engendering Democracy, University Park, Polity Press y Pennsyl- 
vania State University Press, 1991 y de la misma autora Democracy and Difference, University Park, Polity Press y Pennsylvania State University Press, 1993.

${ }^{8}$ El cuestionamiento del concepto de "ciudadano" estriba en que su origen refiere exclusivamente a una persona cuya clase social, sexo y raza están definidos de modo claro: se trata de un hombre blanco, bien educado, parte de la élite y de buena posición económica (algunas autoras añadirán, "heterosexual" para resaltar la importancia de las preferencias sexuales).

${ }^{9}$ Pateman, C., Op. cit. y Phillips, A. (1995), Op. cit.

${ }^{10}$ Véase Lister, Ruth, Citizenship: Feminist Perspectives. Basingstoke, Macmillan, 1997.

${ }^{11}$ Op.cit.

${ }^{12}$ Dietz, Mary G.,"El contexto es lo que cuenta. Feminismo y teorías de la ciudadanía”, en debate feminista, No. 1, marzo 1990, pp. 3-32, original en inglés: Dietz, M., "Context is all: Feminism and theories of citizenship", Daedalus, 116:4, pp.1-24.

${ }^{13}$ Por ejemplo: Gianni, Matteo, "Talking Multiculturalism Seriously: Political Claims for a Differentiated Citizenship", en K. Slawner y M. E. Denham (comps.), Citizenship after Liberalism, Peter Lang, Nueva York, 1998 y Kymlicka, Will, Ciudadanía multicultural, Barcelona, Paidós, 1996.

${ }^{14}$ Yuval-Davis, Nira, "Women, Citizenship and Difference", Feminist Review, No. 57, 1997, pp. 4-27.

${ }^{15}$ Oyekaran, Olasope O. and Michael Olu Adediran "Colonialism, Citizenship and Fractured National Identity: the African Case" en Oommen T.K. (ed.) Citizenship and National Identity: From Colonialism to Globalism. New Delhi, Cloth, 1997, pp. 173-179.

${ }^{16}$ Mudimbe, V. Y. The Invention of Africa: Gnosis, Philosophy, and the Orders of Knowledge. Bloomington, Indiana University Press, 1988, p. 1.

${ }^{17}$ McFadden, Patricia, "Becoming Poscolonial. African Women Changing the Meaning of Citizenship", en $\mathrm{Me}$ ridians: feminism, race, transnationalism, Vol. 6 , no. 1 , 2005, pp. 1-22.

${ }^{18}$ Durante la Cuarta Conferencia Internacional de Mujeres se adoptaron Plan de Acción y la Declaración de
Beijing. Los documentos establecían que el logro de paz, desarrollo e igualdad a nivel local, nacional, regional y global está íntimamente ligado con el avance de las mujeres. En los mismos, además de afirmarse el reconocimiento de los derechos de las mujeres como derechos humanos, se instaba a los gobiernos a tomar medidas para asegurar el igual acceso de las mujeres a todas las esferas de toma de decisiones creando un equilibrio de género en el gobierno y la administración; a integrar a las mujeres en los partidos políticos; a aumentar la capacidad de las mujeres para participar en la toma de decisiones y puestos de liderazgo promoviendo su activo desempeño en los procesos electorales y las actividades políticas. Sobre todo se alentaba la participación de las organizaciones de mujeres como miembros de la sociedad civil y con respeto por su autonomía para implementar el Plan de Acción. Para ello se estableció una agenda que identificaba doce áreas críticas de prioridad de acción para los estados responsables. Así, bajo esta influencia, también en África, las cuestiones sobre las mujeres se ubicaron en torno a la cuestión de los derechos humanos.

${ }^{19}$ Para un análisis más pormenorizado véase Cejas, Mónica I., "Creating a Women's Political Space within the Anti-Apartheid Movement of 1950's: The Case of the Federation of South African Women (1954-1963)", PH. D. Thesis, Tokyo, Tsuda College, Department of International and Cultural Studies, 2003, capítulo II.

${ }^{20}$ Por razones de extensión de este artículo sólo voy a referirme a las africanas, para los casos de otros grupos véase Cejas, Mónica, Creating... op. cit., capítulo 2.

${ }^{21}$ Véase Bradford, Helen “'We are now the men': Women's Beer Protests in the Natal Countryside, 1929", en Bozzoli, Belinda Class, Community and Conflict. South African Perspectives. Johannesburg: Ravan Press, 1987, p. 293.

${ }^{22}$ Idem, p. 303.

${ }^{23}$ Yawitch, Joanne "Tightening the noose: African women and influx control in South Africa 1950-1980", Second Carnegie Inquiry into Poverty and Development in Southern Africa, Conference Paper N. 82. Cape Town, 13-19 April, 1984. 
${ }^{24}$ En efecto, el término "pass" era usado para describir a cualquier documento que se utilizaba para controlar la libertad de movimiento de los africanos y que debía presentarse ante la demanda de las autoridades. Así, los permisos de residencia, los requeridos para entrar a la ciudad, los de búsqueda de empleo. Las principales características de los pases eran las siguientes: sólo las personas de un grupo racial en particular tenía obligación de portarlo; llevaba implícita una restricción a la libertad de movimiento del portador y éste debía llevarlo consigo todo el tiempo ya que su no presentación ante el requerimiento de las autoridades era considerada ofensa punible. Véase Muriel Horrell, ed., Laws Affecting Race Relations in South Africa, 1948-1976, Johannesburg, South African Institute of Race Relations, 1978, p. 175.

${ }^{25}$ Mediante la ley de restauración y extensión de la ciudadanía sudafricana (Restoration and Extension of South African Citizenship Act 196) sancionada en 1993 se aseguró la participación de todos los ciudadanos de los homelands en las primeras elecciones libres, pues se les restituyó la ciudadanía sudafricana, esta vez sí con inclusión del derecho a voto (siguió sin embargo persistiendo la doble ciudadanía pues no se abolieron con esta ley los estatutos que gobernaban a los homelands) Recién con la sanción de la nueva constitución en 1996 se unificó la república y desaparecieron los antiguos homelands reabsorbidos e integrados en nuevas regiones administrativas.

${ }^{26}$ Dicho año 26 agrupaciones políticas se reunieron en Kempton Park, cerca de Johannesburg para elaborar el borrador de la Constitución que habría de poner fin al orden legal de la era del apartheid. Tratándose de grupos no electos, se consideró que dicha Constitución no podría considerarse definitiva, sino más bien, un instrumento de la transición a la vida democrática, de allí su carácter interino hasta que mediante elecciones democráticas se constituyese una Asamblea Constitucional encargada de la redacción de una Constitución definitiva.

${ }^{27}$ Sobre un análisis de la Women's Charter véase Cejas, Mónica, "Apartheid Seen Through Women's Eyes:
The Case of the Federation of South African Women (FSAW) in the 1950's" en Chizuko Tominaga (ed.), Rethinking African History from Women's/Gender Perspectives. JCAS Symposium Series 20, Osaka, National Museum of Ethnology, 2004, pp. 93-11l.

${ }^{28} \mathrm{La}$ Carta de la libertad fue aprobada por unanimidad por el Congreso del Pueblo, celebrado en Kliptown, cerca de Johannesburg, el 25 y 26 de junio de 1955 con lo que pasó a ser el documento fundamental de la lucha contra el apartheid. Véase el texto completo en:

http://www.kairos.org.ar/articuloderevistaiym. php?ID=1283

${ }^{29}$ La Constitución de la República de Sudáfrica (establecida como república en 1966) -Ley 108 de 1996-fue aprobada por la Corte Constitucional el 4 de diciembre de 1996 y entró en vigencia el 4 de febrero de 1997. La Constitución es la ley suprema del país y está por encima de cualquier otra ley o acción del gobierno.

${ }^{30}$ En 1995 fue aprobada la ley de ciudadanía sudafricana (South African Citizenship Act 88) que uniformó las reglas de ciudadanía para todos y en toda la república.

${ }^{31}$ Véase Liebenberg, Sandra, "Social Security and Human Rights: Current Issues", Economic and Social Rights in South Africa Review 1:2, 1998.

${ }^{32}$ Véase el texto completo en http://www.info.gov.za/documents/constitution/index.htm

${ }^{33}$ La plataforma de acción emanada de la IV Conferencia Mundial sobre la Mujer (Pekín, 1995), condujo a la adopción del concepto de gender mainstreaming o "integración sistemática de la dimensión de igualdad de oportunidades". Definida como estrategia -no un fin en sí misma sino un medio- para promover la igualdad de género, gender mainstreaming implicaría la consideración sistemática de las diferencias entre las condiciones, las situaciones y las necesidades de mujeres y hombres, en el conjunto de las políticas y de las actuaciones de los poderes públicos. De acuerdo a Naciones Unidas, esto no implica el desarrollo de proyectos para mujeres dentro de programas, ni tampoco agregar componentes "femeninos" en los programas de trabajo existentes; sino 
que requiere que se dé atención a las perspectivas de género como parte integral de todas las actividades, es decir, volverlas transversales a todos los programas.

${ }^{34}$ A nivel provincial se implementaron en 8 de las 9 provincias.

${ }^{35}$ De acuerdo a Seidman-Makgetla, en 2003 el $96 \%$ de las trabajadoras domésticas eran mujeres de color (africanas, de origen indio y colored). 93\% de ellas percibían un salario mensual menor a los 1,000 rands (entre 400 y 450 pesos argentinos). Seidman-Makgetla, Neva, "Women and the Economy in South Africa", 2004, pp. 2 y 7 .

http:/www.genderstats.org.za/economy.shtml

${ }^{36}$ Idem, p. 15.

${ }^{37}$ Véase Walker, Cherryl, "Land Reform and Gender in Post-Apartheid South Africa", UNRISD Discussion Paper No. 98, October 1998.

${ }^{38}$ Walker, Cherryl, "Agrarian Change, Gender and Land Reform. A South African Case”, UNRISD Social Policy and Development Programme Paper Number 10 April 2002.

${ }^{39}$ Véase Hassim, Shireen, "Turning Gender Rights into Entitlements: Women and Welfare Provisions in Postapartheid South Africa", Social Research, Fall 2005.

40 "State of the Nation Address of the President of South Africa..." Op.cit.

41 "...that is ugly and repulsive in human society".

${ }^{42}$ Op. cit. (la traducción al español es mía, puede verse la versión original en inglés en el sitio en internet ya señalado).

${ }^{43}$ En este sentido tanto la censura, como la segregación (incluyendo el caso extremo del apartheid), la coerción económica, la tortura, la represión, han sido y son medios para asegurar la representación de la nación como una comunidad, la unidad que para ser imaginada necesita excluir, diferenciar, instituir a un/unos otros. Algunas formas identitarias como las sexualidades consideradas impropias son excluidas produciendo lo que Teresa de Laurentis ha llamado "socio-sexual (in) diferencia" en
De Laurentis, Teresa, "Sexual Indifference and Lesbian Representation”, en Theater Journal, 40, 2 (mayo 1988), pp. 151-177.

${ }^{44}$ Pedagogía de la nación que de acuerdo a Bhabha tiene por objeto producir y disciplinar ciudadanos-sujetos. Véase Bhabha, Homi K., Nation and Narration. London, Routledge, 1990.

45 Aludiendo aquí al anquilosamiento que caracterizaría a las instituciones que deberían garantizar políticas transversales de género como la Comisión para la igualdad de género (CGE por sus siglas en inglés). Esta última ha sido duramente criticada en un informe de la Unión Europea hecho público en febrero de este año, según el cual la Comisión "habla mucho pero hace poco... se ha aislado de la sociedad civil, se ha cerrado al debate, incapaz de comprometerse con una agenda feminista transformadora y muestra una actitud irresponsable en el amplio espectro de cuestiones que impactan a la igualdad de género y las vidas de las mujeres en Sudáfrica". Véase Lynley Donnelly y Fikile-Ntsikelelo Moya, "Reports slam gender watchdog" en Mail \& Guardian, 23 de febrero de 2007.

${ }^{46}$ El énfasis en negritas es mío.

\section{Bibliografía \\ Documentos}

Department of Foreign Affairs Republic of South Africa, "State of the Nation Address of the President of South Africa, Thabo Mbeki: Joint Sitting of Parliament, February 9, 2007", en http:/www.dfa.gov.za/docs/ speeches/2007/mbek0209.htm.

GENTA, "A Feminist critique of president Mbeki", Pambazuka News, 14-02-2007, en http://www.pambazuka. org/en/category/comment/39753. South African Government, Constitution of the Republic of South Africa, 1996 http://www.info.gov.za/documents/constitution/ index.htm

Statistics South Africa, 2003, Labour Force Survey, Pretoria. 


\section{Fuentes secundarias}

Bhabha, Homi K., 1990, Nation and Narration. London, Routledge.

Bradford, Helen, 1929, “'We are now the men”: Women's Beer Protests in the Natal Countryside,' in Bozzoli, Belinda (ed.) Class, Community and Conflict. South African Perspectives, Johannesburg: Ravan Press, 1987, pp. 292323.

Cejas, Mónica I., 2003, "Creating a Women's Political Space within the Anti-Apartheid Movement of 1950's: The Case of the Federation of South African Women (1954-1963)", PH. D. Thesis, Tokyo, Tsuda College, Department of International and Cultural Studies.

Cejas, Mónica, 2004, “Apartheid Seen Through Women's Eyes: The Case of the Federation of South African Women (FSAW) in the 1950's" en Chizuko Tominaga (ed.), Rethinking African History from Women's/Gender Perspectives. JCAS Symposium Series 20, Osaka, National Museum of Ethnology, pp. 93-111.

De Laurentis, Teresa, 1988, "Sexual Indifference and Lesbian Representation", en Theater Journal, 40, 2, pp. 151-177.

Dietz, Mary G., 1990, "El contexto es lo que cuenta. Feminismo y teorías de la ciudadanía", en debate feminista, No. 1, pp. 3-32, original en inglés: Dietz, M., "Context is all: Feminism and theories of citizenship", Daedalus, 116:4, pp.1-24.

Donnelly, Lynley y Fikile-Ntsikelelo Moya, 2007, "Reports slam gender watchdog” en Mail $\backsim$ Guardian.

Gianni, Matteo, 1998, "Talking Multiculturalism Seriously: Political Claims for a Differentiated Citizenship", en K. Slawner y M. E. Denham (comps.), Citizenship after Liberalism, Peter Lang, Nueva York.

Hassim, Shireen, 2005, "Turning Gender Rights into Entitlements: Women and Welfare Provisions in Postapartheid South Africa", Social Research, Fall.

Horrell, Muriel ed., 1978, Laws Affecting Race Relations in South Africa, 1948-1976, Johannesburg, South African Institute of Race Relations.
Jelin, Elizabeth, 1997, "Igualdad y diferencia: dilemas de la ciudadanía de las mujeres en América Latina”, Ágora, No. 7, Año 3, pp. 189-213.

Jelin, Elizabeth, 1996, Las mujeres y la cultura ciudadana en América Latina, UBA-CONICET, mímeo, Buenos Aires.

Kymlicka, Will, 1996, Ciudadanía multicultural, Paidós, Barcelona.

Liebenberg, Sandra, 1998, "Social Security and Human Rights: Current Issues", en Economic and Social Rights in South Africa Review 1:2.

Lister, Ruth, 1997, Citizenship: Feminist Perspectives. Basingstoke, Macmillan.

McFadden, Patricia, 2005, "Becoming Poscolonial. African Women Changing the Meaning of Citizenship", en Meridians: feminism, race, transnationalism, vol. 6, núm. 1,pp. 1-22.

Mudimbe, V. Y., 1988, The Invention of Africa: Gnosis, Philosophy, and the Orders of Knowledge, Bloomington, Indiana University Press.

Oyekaran, Olasope O. and Michael Olu Adediran, 1997, "Colonialism, Citizenship and Fractured National Identity: the African Case", en Oommen T.K. (ed.) Citizenship and National Identity: From Colonialism to Globalism. New Delhi, Cloth, pp. 173-179.

Pateman, Carole, 1989, The Disorder of Women, Stanford University Press, Stanford.

Phillips, Anne, 1991, Engendering Democracy, University Park, Polity Press y Pennsylvania State University Press.

Phillips, Anne, 1993, Democracy and Difference, University Park, Polity Press y Pennsylvania State University Press.

Phillips, Anne, 1995, The Politics of Presence, Oxford, Clarendon Press.

Reitzes, M., 1995, "Insiders and Outsiders: The Reconstruction of Citizenship in South Africa" Policy:Issues and Actors, Vol. 8, n.l Johannesburg, Centre for Policy Studies.

Ricci, François, 1977, Gramsci dans le texte. Paris, Editions Sociales. 
Seidman-Makgetla, 2004, Neva, "Women and the Economy in South Africa", en http://www.genderstats. org.za/economy.shtml.

Sunder Rajan, Rajeshwari, 2003, The Scandal of the State: Women, Law, and Citizenship in Postcolonial India. Next Wave: New Directions in Women's Studies Series. Durham and London: Duke University Press.

Walker, Cherryl, 1998, "Land Reform and Gender in Post-Apartheid South Africa", en UNRISD Discussion Paper núm. 98.

Walker, Cherryl, 2002, "Agrarian Change, Gender and Land Reform. A South African Case", en UNRISD
Social Policy and Development Programme Paper núm. 10.

Yawitch, Joanne, 1984, "Tightening the noose: African women and influx control in South Africa 1950-1980", Second Carnegie Inquiry into Poverty and Development in Southern Africa, Conference Paper núm. 82. Cape Town, 13-19.

Yuval-Davis, Nira \& Pnina Werbner, 1999, Women, Citizenship and Difference, London, Zed Books.

Young, Iris Marion, 1989, "Polity and Group Difference: A Critique of the Ideal of Universal Citizenship", en Ethics, 99, núm. 2, pp. 250-274. 